\title{
Caffeine use in preterm infants: from magic to uncertainty
}

\begin{abstract}
Caffeine is one of the most commonly used drugs in neonatology. Basically, it is indicated for preterm infants with apnea, especially for those born at less than 28 weeks of gestation. However, there is serious uncertainty regarding the time for starting and ending its use and its eventual benefits on neurodevelopment. Possibly, some of these benefits are related to a shorter use of mechanical ventilation and apnea events, which would directly result in a lower incidence of bronchopulmonary dysplasia (BPD), which is another potential indication for this intervention. ${ }^{1}$

Oxygen fluctuations (intermittent and recurrent hypoxemia) secondary to apnea, which are more common at a younger gestational age, have been related to adverse events such as retinopathy of prematurity, growth and neurodevelopmental disorders, and mortality. ${ }^{2}$ Most likely, the benefits of caffeine are not related to a direct impact on lung and neurological function, but rather to apnea prevention. However, it is necessary to establish if there are other mechanisms involved.
\end{abstract}

Based on this, withdrawing invasive/noninvasive mechanical ventilation and maintaining a respiratory pattern with the lowest number of apnea events possible is a priority in this group of preterm infants. Multiple therapeutic alternatives have been used to this end, such as non-invasive ventilation and xanthines. Among the latter, caffeine is the first option due to its pharmacological advantages and its promotion by the Caffeine for Apnea of Prematurity (CAP) Trial Group. One of the main conclusions of that study was that preterm infants born at less than 28 weeks of gestation receiving caffeine early (at less than 3 days) had a lower incidence of BPD compared to the non-intervention group. This outcome was not part of the study's main objective but it was observed that no apnea events were reported in the target population and that events were less serious in the caffeine group. ${ }^{3}$

Since then, caffeine has positioned itself as the first pharmacological choice for apnea management and BPD prevention; in addition, it is considered an option to successfully withdraw mechanical ventilation in the population at a higher risk for apnea. This has promoted its use in an earlier manner but there is uncertainty regarding its use for the prevention of apnea, especially in relation to the adequate time for indication.

Recent studies have raised even more questions. ${ }^{4}$ On the one side, early caffeine use, looking to achieve a successful extubation and also a lower BPD incidence, has not demonstrated improved outcomes. On the other side, the study was interrupted due to a higher mortality, which was above the intervention site's historical numbers. Other authors have already reported data that confirmed not only that caffeine failed to facilitate extubation and to reduce the length of mechanical ventilation use in small preterm infants, which is not unusual due to the poor respiratory effort at this gestational age, but also that mortality increased in association with an early caffeine use. ${ }^{2}$

It is expected that some preterm infants, especially those with a birth weight of less than $750 \mathrm{~g}$, born at less than 26 weeks of gestation, and with an alveolar-arterial oxygen gradient $>$ $180 \mathrm{mmHg}$, have a higher risk for failure with continuous positive airway pressure (CPAP). ${ }^{5}$ These risk factors also probably determine a failure for the implementation of early caffeine use. This has also been reported in preterm infants with a birth weight of less than $1500 \mathrm{~g}$; the success of CPAP during the first week of life in patients for whom early caffeine was indicated has not been demonstrated. ${ }^{6}$ The indication for its early use should be reviewed and, most likely, during its use, it may be necessary to assess the patient's clinical condition.

As mentioned above, recent studies have associated caffeine early use with an increased mortality, so caffeine interventions should be managed very cautiously. In addition, certain cohorts (relative risk [RR]: 0.80, $95 \%$ confidence interval [CI]: 0.66-0.96) and controlled clinical trials (RR: $0.67,95 \%$ CI: 0.56-0.81) were studied in a meta-analysis, and it was observed that an early initiation was related to a reduction in BPD. In cohort studies, other benefits included a reduction in patent ductus arteriosus, brain injury, and retinopathy of prematurity, and a lower use of postnatal corticosteroids. However, in the same meta-analysis, mortality increased. ${ }^{7}$

Other harmful effects of caffeine use are now being explored, such as a possible association with osteopenia of prematurity. This would 
be directly related to the length of use and the accumulated caffeine dose. The dilemma has not been resolved yet, especially in relation to the time of initiation and use, in addition to establishing the optimal dose and other potential benefits, such as a direct neurological impact. ${ }^{8}$

Besides, a new question arises about whether higher doses would be more effective to prevent apnea and BPD. However, adverse events such as an impact on astrocyte generation and cerebellar involvement have been reported. Consequently, trying to increase the dose in an attempt to reduce apnea periods should not become a routine practice. $^{9}$

The uncertainty caused by the intervention in terms of its actual impact remain unsolved from a clinical standpoint. However, its increased use in preterm infants has become widespread, for example, as apnea prophylaxis, for which there is no current appropriate evidence.

Caffeine is the first choice for the management of apnea of prematurity with favorable outcomes in relation to the management of apnea and the reduction of BPD, patent ductus arteriosus, and retinopathy of prematurity.

It is necessary to determine whether the molecule's properties will have a direct benefit on neuroprotection, which has been hypothetically reported in animal studies that showed neuroprotection at high oxygen levels and against periventricular injury. But harmful effects have also been reported, such as the negative effect on the alveolarization and apoptosis of type II pneumocytes. ${ }^{1,10}$

Such contradictory results have generated uncertainty in relation to the intervention's additional benefits. Caffeine has clear benefits, and certain trends may have been accepted:

1. There is no strong evidence in favor of caffeine use as prophylaxis of apnea of prematurity.

2. Early caffeine use should be individualized because there will probably be patients in whom the intervention will not achieve a successful mechanical ventilation withdrawal.

3. High caffeine doses are not recommended due to potential neurological damage.

4. Therapy duration will be determined based on adjusted gestational age; most likely, immature preterm infants will require therapy for a longer period.
5. In addition, it is recommended to assess withdrawal for approximately one week once no apnea events are registered in the preterm infant.

6. Routine measurement of levels is not necessary when using usual doses.

Jorge L. Alvarado Socarras M.D. Coordinator of the Neonatal Care Unit of Fundación Cardiovascular de Colombia.

Latin American Organization for Health Research Promotion (Organización Latinoamericana para el Fomento de la Investigación en Salud, OLFIS), Colombia.

Jorgealso2@yahoo.com

http: / / dx.doi.org/10.5546/ aap.2018.eng.380

To cite: Alvarado Socarras JL. Caffeine use in preterm infants: from magic to uncertainty. Arch Argent Pediatr 2018;116(6):380381.

\section{REFERENCES}

1. Abu-Shaweesh JM, Martin RJ. Caffeine use in the neonatal intensive care unit. Semin Fetal Neonatal Med 2017; 22(5):342-7.

2. GentleSJ, Travers CP, Carlo WA. Caffeine controversies. Curr Opin Pediatr 2018; 30(2):177-81.

3. Schmidt B, Roberts RS, Davis P, et al. Caffeine therapy for apnea of prematurity. N Engl J Med 2006; 354(20):2112-21.

4. Amaro CM, Bello JA, Jain D, et al. Early Caffeine and Weaning from Mechanical Ventilation in Preterm Infants: A Randomized, Placebo-Controlled Trial. J Pediatr 2018; 196:52-7.

5. O'Donnell CP, Stenson BJ. Respiratory strategies for preterm infants at birth. Semin Fetal Neonatal Med 2008; 13(6):401-9.

6. Patel RM, Zimmerman K, Carlton DP, et al. Early Caffeine Prophylaxis and Risk of Failure of Initial Continuous Positive Airway Pressure in Very Low Birth Weight Infants. J Pediatr 2017; 190:108-11.e1.

7. Kua KP, Lee SW. Systematic review and meta-analysis of clinical outcomes of early caffeine therapy in preterm neonates. Br J Clin Pharmacol 2017; 83(1):180-91.

8. Ali E, Rockman-Greenberg C, Moffatt M, et al. Caffeine is a risk factor for osteopenia of prematurity in preterm infants: a cohort study. BMC Pediatr 2018; 18(1):9.

9. Dobson NR, Patel RM. The Role of Caffeine in Noninvasive Respiratory Support. Clin Perinatol2016; 43(4):773-82.

10. NobileS, Carnielli VP. Caffeine for preterm infants: current indications and uncertainties. Acta Biomed 2015; 86 (Suppl 1):32-5. 